\title{
Modified Vertically Installed Planar Couplers for Ultrabroadband Multisection Quadrature Hybrid
}

\author{
Hui-Chun Chen and Chi-Yang Chang, Member, IEEE
}

\begin{abstract}
In this letter, we describe a five-section ultrabroadband 1-9 GHz microstrip quadrature hybrid $\left(90^{\circ},-3 \mathrm{~dB}\right.$ coupler $)$, which uses two types of modified vertically installed planar (VIP) couplers and is suitable for conventional printed circuit board realization. The modified VIP couplers provide not only a way to implement tight coupling value with reduced modal phase velocity difference but also a layout flexibility to minimize the discontinuity between sections. An experimental circuit is designed and fabricated with a passband ripple of $\pm 0.5 \mathrm{~dB}$, a return loss of less than $-20 \mathrm{~dB}$, and an isolation of better than $20 \mathrm{~dB}$ over the frequency of 1-9 GHz. Very good agreement between the simulated and measured results is obtained.
\end{abstract}

Index Terms-Modified vertically installed planar (VIP) coupler, multisection hybrid, quadrature hybrid, VIP coupled line.

\section{INTRODUCTION}

Q UADRATURE hybrid is one of the key elements in many microwave circuits such as a balanced amplifier, a balanced mixer, a phase shifter, a power divider, and a beamforming network for array antennas and for direction-finding antennas. Nowadays, ultrabroadband performance with a low cost printed circuit board (PCB) process are demanded in many applications. A single-section vertically installed planar (VIP) quadrature hybrid using the PCB process has been demonstrated in [1], [2] but none of the multisection quadrature hybrids using the PCB process has been reported. The most difficult issues in realizing a multisection quadrature hybrid are achieving an extremely tight-coupled center section, equalizing modal phase velocities, and minimizing the discontinuity effect between each section. In the past, only strip line circuits can solve all three of these problems. Unfortunately, unlike microstrip circuits, the conventional stripline circuits need a complex metal housing. For microstrip implementation, the Lange [3] coupler using high dielectric constant substrate can achieve tight coupling of about $-3 \mathrm{~dB}$ but it is not suitable for the PCB process (due to the PCB's low dielectric constant substrate) and could not achieve the required $-0.8 \mathrm{~dB}$ of coupling. Besides, a multisection quadrature hybrid using a Lange coupler and parallel-coupled lines encounters a serious discontinuity problem at the junction between each section. The VIP coupler in [2] solves the problem of PCB realization of tight coupled lines, but their modal phase velocities are not compensated and, according to our analysis, the structure in

Manuscript received March 6, 2006; revised May 2, 2006. This work was supported in part by the National Science Council of Taiwan, R.O.C. under Grant NSC94-2752-E-09-003-PAE.

The authors are with the Department of Communication Engineering, National Chiao-Tung University, Hsinchu 300, Taiwan, R.O.C. (e-mail: andrew@gemtek.com.tw; mhchang@cc.nctu.edu.tw).

Digital Object Identifier 10.1109/LMWC.2006.879491

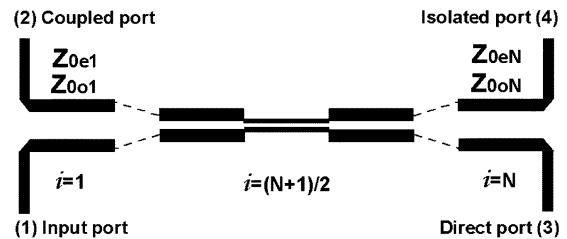

Fig. 1. $N$-section quadrature hybrid.

[2] can not realize a coupler with coupling as tight as $-0.8 \mathrm{~dB}$. Unequal modal phase velocities have little influence on the performances of a single-section quadrature hybrid but seriously degrade the performances of a multisection quadrature hybrid. Many studies [4]-[8] have been conducted to compensate the modal phase velocities of a coupler. However, none of them are suitable for the VIP coupler. In this letter, we propose a five-section ultrabroadband quadrature hybrid, which is suitable for PCB realization. The two outer sections use conventional microstrip parallel-coupled lines and the three inner sections comprise two types of modified VIP couplers where one of them provides extra layout flexibility for minimizing the discontinuity between each section. Moreover, special dielectric blocks are proposed to equalize the modal phase velocities of the modified VIP couplers.

\section{Design CuRves}

A multisection quadrature hybrid is a symmetric circuit that the $i$ th section is identical to the $(N+1-i)$ th section as shown in Fig. 1. Each section is a quarter wavelength long at center frequency. In this letter, a five-section quadrature hybrid is demonstrated as an example. For the loosely coupled outmost sections (sections 1 and 5), conventional microstrip parallel-coupled line couplers are used which can be easily synthesized using any commercial circuit simulator.

For the second and fourth sections, a type I modified VIP coupler is proposed as shown in Fig. 2(a). The dielectric blocks at both sides of the VIP coupler, which use similar material as the main and VIP substrate, increase the effective dielectric constant of even-mode. In Fig. 2(a), there are four metal strips of which two are on the VIP substrate and the other two are on the main substrate. The strips on the VIP substrate and the strips on the main substrate are connected at two ends and at the center of the coupler. This structure can implement a coupler with coupling from moderate to tight coupling. The even- and odd-mode characteristic impedances with respect to the VIP metal height $\left(H_{\text {metal }}\right)$ and the width of the strips on the main substrate $(\mathrm{W})$ are extracted by the EM simulation tool HFSS. Fig. 3(a)-(b) depicts the extracted data, in which the gap width between two strips on the main substrate $(G)$ is 18 and 28 mils, respectively. 
(a)

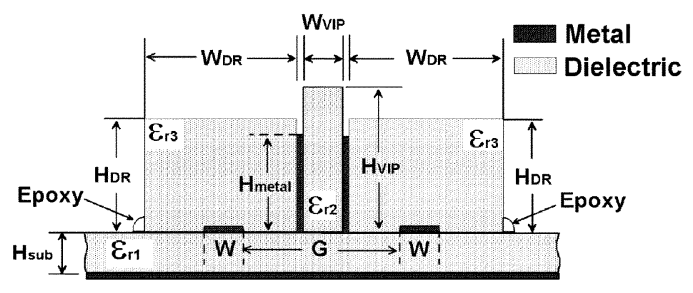

(b)

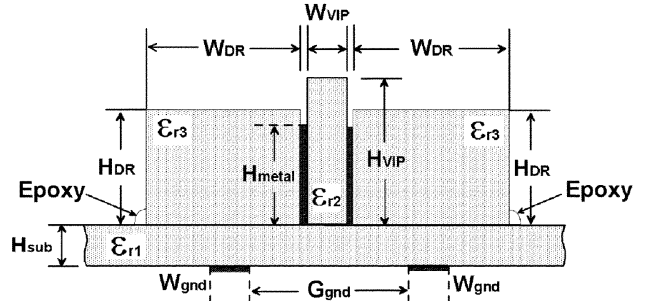

Fig. 2. Cross-sectional views of (a) type I, and (b) type II of the modified VIP couplers, where $H_{\text {sub }}=20$ mils, $W_{\mathrm{VIP}}=8 \mathrm{mils}, H_{\mathrm{VIP}}=120 \mathrm{mils}, W_{\mathrm{DR}}=$ 200 mils, $W_{\text {gnd }}=15$ mils, $H_{\mathrm{DR}}=60$ mils, and $\varepsilon_{\mathrm{r} 1}=\varepsilon_{\mathrm{r} 2}=\varepsilon_{\mathrm{r} 3}=3.38$.
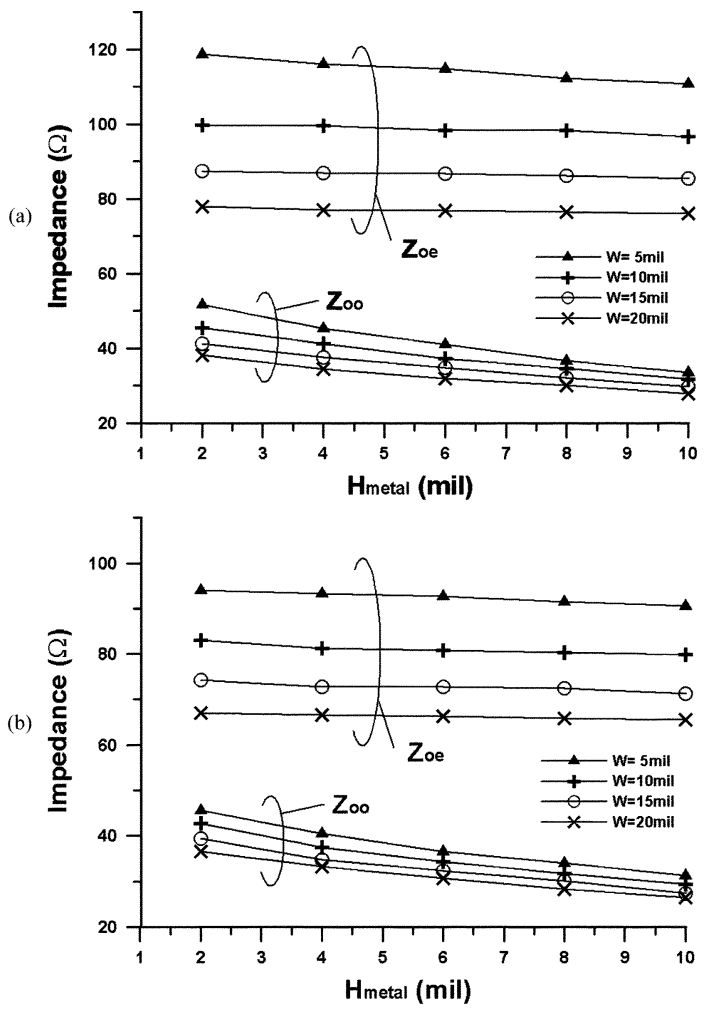

Fig. 3. Even- and odd-mode characteristic impedances versus VIP metal height ( $H_{\text {metal }}$ ) of the type I VIP coupler with (a) $G=18$ mils and (b) $G=82$ mils.

Observing these curves, the even-mode impedance is mainly decided by the width of the strips on the main substrate $(\mathrm{W})$ while the odd-mode characteristic impedance is mostly controlled by the VIP metal height $\left(H_{\text {metal }}\right)$. The gap $G$ provides another degree of freedom to choose the total width of the coupler so that the junctions connecting to other sections can be laid out with minimal discontinuity.

The type I of modified VIP coupler can not achieve a coupling value as tight as $-0.8 \mathrm{~dB}$ even when $W$ equals 0 . To implement the extremely tight-coupled center section, the type II of modified VIP coupler is proposed as shown in Fig. 2(b). In Fig. 2(b), the ground plane in the main substrate changes to two metal strips. Utilizing this finite-extent ground plane (in
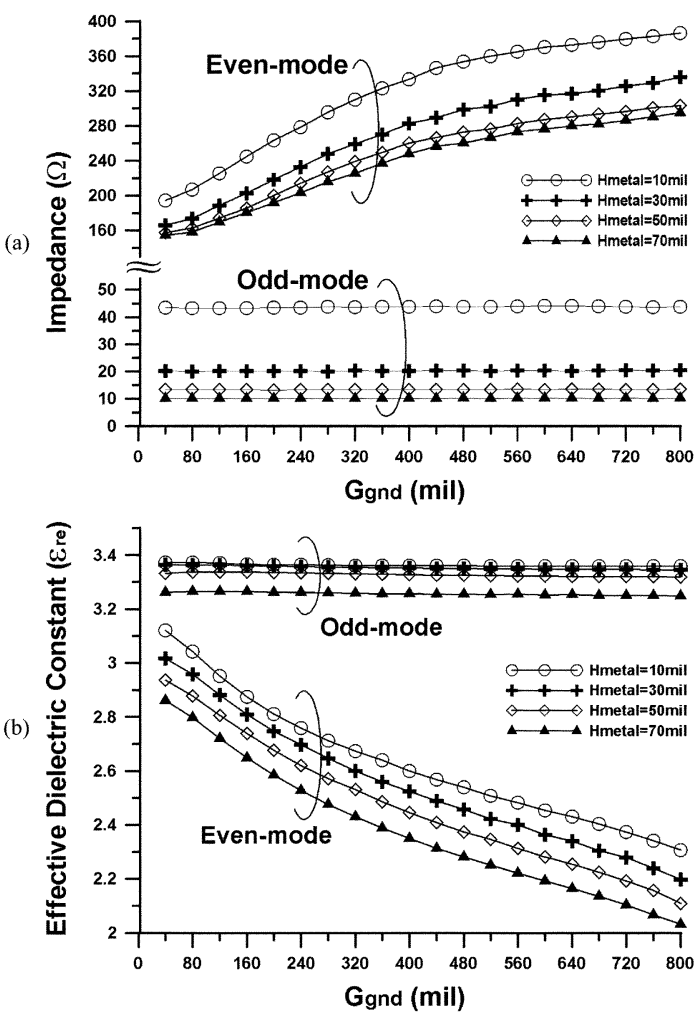

Fig. 4. (a) Even- and odd-mode characteristic impedances and (b) effective dielectric constants versus $G_{\text {gnd }}$ and $H_{\text {metal }}$ of the type II VIP coupler.

TABLE I

Design Parameters and Physical Dimensions OF EXPERIMENTAL COUPLER

\begin{tabular}{|c|c|c|c|c|c|c|c|c|c|c|}
\hline \multicolumn{6}{|c|}{ Coupler Specifications } & \multicolumn{5}{|c|}{ Dimension (mm) } \\
\hline Freq & $\begin{array}{cc}\mathrm{C} \\
(\mathrm{dB})\end{array}$ & Ripple & Section & $Z_{0 \mathrm{e}}$ & $Z_{0 o}$ & W & G & $\mathrm{H}_{\text {metal }}$ & $\mathrm{G}_{\mathrm{gnd}}$ & $\mathrm{L}$ \\
\hline \multirow{3}{*}{$1-9 \mathrm{GHz}$} & \multirow{3}{*}{$3 \mathrm{~dB}$} & \multirow{3}{*}{$+/-0.5 \mathrm{~dB}$} & $\mathrm{I}=1,5$ & 58.8 & 42.5 & 1.1 & 0.38 & NA & NA & 9.0 \\
\hline & & & $\mathrm{I}=2,4$ & 78.5 & 31.8 & 0.4 & 0.6 & 0.16 & NA & 8.0 \\
\hline & & & $\mathrm{I}=3$ & 233 & 0.7 & NA & NA & 1.5 & 6.6 & 8.0 \\
\hline
\end{tabular}

this case only two ground strips) structure, the VIP coupler can achieve a coupling tighter than $-0.8 \mathrm{~dB}$. Again, a similar dielectric block as the type $\mathrm{I}$ is used to compensate the modal phase velocities. The even- and odd-mode characteristic impedances versus various VIP metal heights $\left(H_{\text {metal }}\right)$ and ground strip spacing $\left(G_{\text {gnd }}\right)$ are shown in Fig. 4(a). Similarly, $G_{\text {gnd }}$ and $H_{\text {metal }}$ separately control the even- and odd-mode characteristic impedances $\left(Z_{0 \mathrm{e}}, Z_{0 \mathrm{o}}\right)$. Here, the width of the ground plan strips $\left(W_{\text {gnd }}\right)$ is chosen to be 15 mils for simplifying the design. Depicted in Fig. 4(b) are the even- and odd-mode effective dielectric constant versus different $H_{\text {metal }}$ and $G_{\text {gnd }}$. With the help of dielectric blocks, the differences between even- and odd-mode effective dielectric constant are minimized.

\section{Circuit Realization and Measurement}

In this letter, a quadrature hybrid with a passband ripple of $\pm 0.5 \mathrm{~dB}$ and frequency range of $1-9 \mathrm{GHz}$ is demonstrated. According to the design table listed in [9], five sections are needed to fulfill such specifications and the corresponding even- and odd-mode characteristic impedances of each section are detailed in Table I. The following design sequence is suggested. First, for the first and fifth sections, use a circuit simulator to get physical 


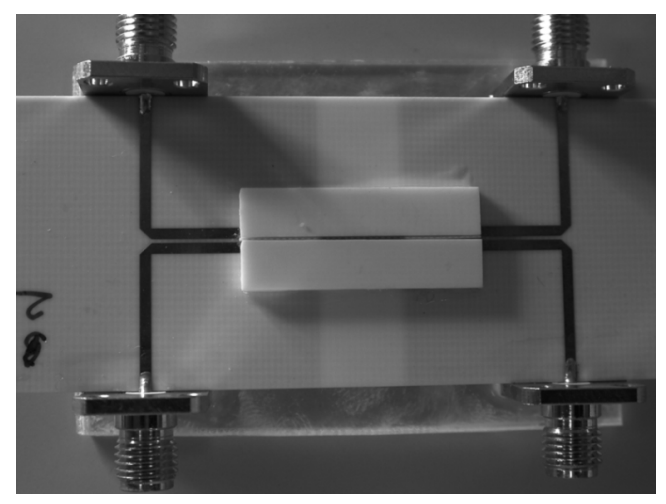

Fig. 5. Photograph of the fabricated five-section 3-dB directional coupler.

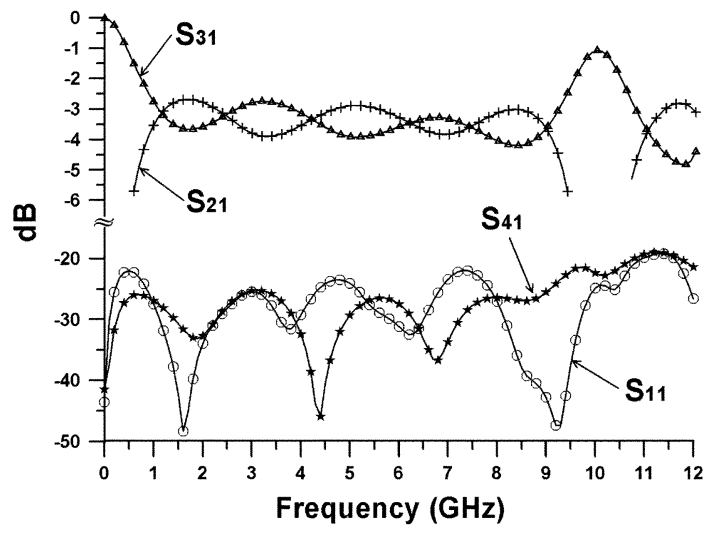

Fig. 6. Simulated responses of the proposed hybrid.

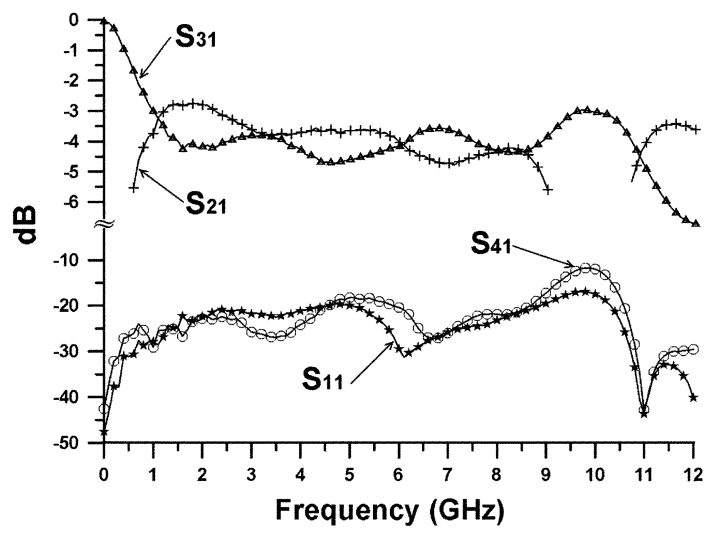

Fig. 7. Measured responses of the proposed hybrid.

dimensions. Then, use Fig. 4(a) and (b) to obtain the physical dimensions of section 3. Last, to design sections 2 and 4, designers should properly choose the width and distance of the strips on the main substrate by the aid of Fig. 3(a) and (b) to minimize the discontinuities between sections (especially between sections 1 and 2 (also 4 and 5). Finally, the whole quadrature hybrid performances are simulated by three-dimensional (3-D) EM simulator HFSS. The physical dimensions of each section are shown in Table I.

Based on the above procedures, the proposed five-section hybrid is fabricated. The photograph of the proposed five-section
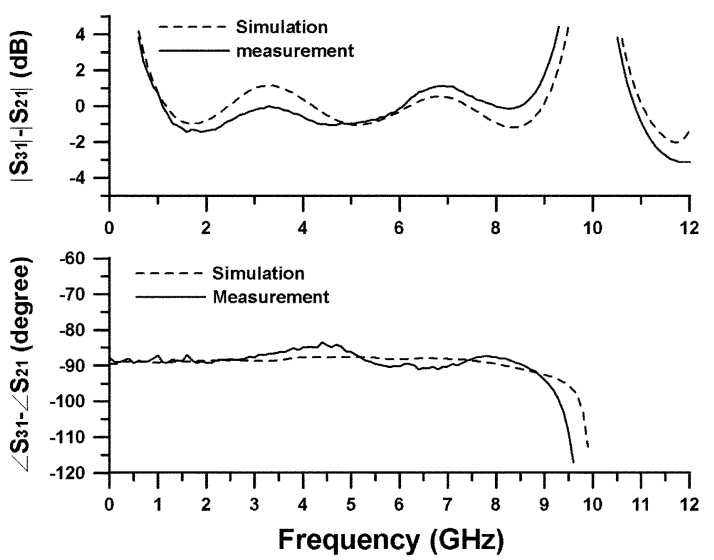

Fig. 8. Measured and simulate amplitude and phase errors of the proposed hybrid.

hybrid is shown in Fig. 5. The main substrate, VIP substrate, and dielectric blocks are all Rogers RO4003 with a dielectric constant of 3.38 and thickness of 8,20 , and 60 mils, respectively.

Depicted in Figs. 6 and 7 are the simulated and the measured results that match well with what could be observed. The simulated and measured amplitude and phase errors are shown in Fig. 8. The measured amplitude and phase balances between port 2 (coupled port) and port 3 (through port) are described as follows. The amplitude difference is less than $\pm 0.65 \mathrm{~dB}$ and the phase difference is keeping at $90 \pm 5^{\circ}$ over the designed frequency of $1-9 \mathrm{GHz}$.

\section{CONCLUSION}

In this letter, a multisection 3-dB quadrature hybrid realized by conventional PCB process has been successfully demonstrated. The proposed modified VIP couplers have solved all three of the problems that a multisection 3-dB quadrature hybrid has always encountered.

\section{REFERENCES}

[1] Y. Konishi, I. Awai, Y. Fukuoka, and M. Nakajima, "A directional coupler of a vertically installed planar circuit structure," IEEE Trans. Microw. Theory Tech., vol. MTT-36, no. 6, pp. 1057-1063, Jun. 1988.

[2] C. Zhao and I. Awai, "Application of the finite difference techniques to the compensated VIP $3 \mathrm{~dB}$ directional coupler," IEEE Trans. Microw. Theory Tech., vol. 44, no. 11, pp. 2045-2052, Nov. 1996.

[3] J. Lange, "Interdigitated stripline quadrature hybrid," in IEEE MTT-S Int. Dig., May 1969, vol. 69, no. 1, pp. 10-13.

[4] S. L. March, "Phase velocity compensation in parallel-coupled microstrip," in IEEE MTT-S Int. Dig., Jun. 1982, vol. 82, no. 1, pp. 410-412.

[5] S. B. Cohn, "The re-entrant cross section and wide-band 3-dB hybrid couplers," IEEE Trans. Microw. Theory Tech., vol. MTT-11, no. 4, pp. 254-258, Jul. 1963.

[6] B. Sheleg and B. E. Spielman, "Broad-band directional couplers using microstrip with dielectric overlays," IEEE Trans. Microw. Theory Tech., vol. MTT-22, no. 12, pp. 1216-1220, Dec. 1974.

[7] C. Buntschuh, "High directivity microstrip couplers using dielectric overlays," in IEEE MTT-S Int. Dig., May 1975, vol. 75, no. 1, pp. $125-128$.

[8] J. L. Klein and K. Chang, "Optimum dielectric overlay thickness for equal even- and odd- mode phase velocities in coupled microstrip circuits," Electron. Lett., vol. 26, pp. 274-276, 1990.

[9] E. G. Cristal and L. Young, "Theory and tables of optimum symmetrical tem-mode coupled transmission line directional couplers," IEEE Trans. Microw. Theory Tech., vol. MTT-13, no. 5, pp. 544-558, Sep. 1965. 